\section{Combined 20- and 23-gauge pars plana vitrectomy}

\author{
Vitrectomia via pars plana com acesso combinado \\ de 20 e 23 gauge
}

Dear Editor:

The 23-gauge (G) transconjunctival sutureless vitrectomy (TSV) technique has advantages of decreased surgical trauma, decreased astigmatism, faster wound healing, and improved postoperative comfort ${ }^{(1)}$ compared with other vitrectomy techniques. Although the efficacy of 23-G is comparable with conventional 20-G vitrectomy, some surgeons still use 20-G vitrectomy in complicated cases, such

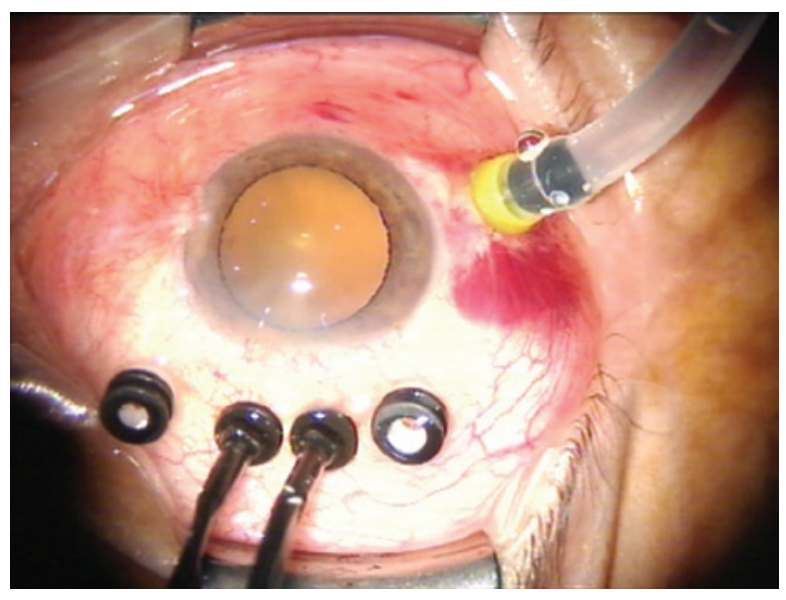

Figure 1. One inferotemporal 23-G TSV cannula for infusions and two superior 20-G one-step TSV cannulae with internal valves to minimize leakage. as complex retinal detachments $(R D)^{(2)}$. Herein, we describe a modified technique combining 20- and 23-G sclerotomies and the use of 20-G instruments that provides the advantages of both techniques in a single procedure.

Combined surgery was performed in 67 eyes of 65 patients by a single surgeon. During the follow-up period, anatomical and functional outcomes and operative complications were evaluated. Postoperative vitreous hemorrhage $(\mathrm{VH})$ was classified into two groups; early postoperative $\mathrm{VH}$ (occurring within 1 month postoperatively) and late postoperative $\mathrm{VH}$ (occurring $>1$ month postoperatively). Transient postoperative hypotony was defined as intraocular pressure (IOP) $<6 \mathrm{mmHg}$ lasting $<1$ week. The degree of intraocular inflammation was clinically defined by the presence of transient fibrin formation in the anterior chamber or vitreous cavity.

One inferotemporal 23-G transconjunctival sutureless sclerotomy port for infusion and two superior 20-G sclerotomy ports for instrumentation, including a vitrectomy probe, were created along with two 25-G Torpedo minilight illuminations bimanual vitrectomy. A 23-G single-step TSV trocar-cannula (EyeTech) was transconjunctivally inserted at a $30^{\circ}$ angle inferotemporally to the infusion line in all cases. The two superior 20-G sclerotomy ports were created with a $20-\mathrm{G}$ one-step preloaded trocar cannula at a $30^{\circ}$ angle tangential to sclera (Synergetic. Inc, Figure 1). The internal valves of the cannula minimize leakage and aid in the maintenance of IOP during the surgical procedure. After completing the vitrectomy, a cotton-tipped applicator was applied to the trocar entry site to prevent leakage from the sclerotomy during the removal of the trocars. The trocar entry site was inspected for wound leakage and then closed with a single 8-0 Vicryl suture.

The mean age of study participants was $63.4 \pm 14.2$ years and 35 (54.6\%) were male. The median follow-up period was 3 months (range, 1-6 months). Best corrected visual acuity increased in 39 eyes (58.2\%), remained unchanged in 25 eyes (37.3\%), and decreased in three eyes (4.4\%) because of cataract progression in two eyes and intraoperative choroidal detachment in one eye. Surgical parameters are presented in table 1.

Table 1. Surgical parameters

\begin{tabular}{|c|c|c|c|c|c|}
\hline Indications for surgery & Number of eyes & Preoperative BCVA (logMAR) & Postoperative BCVA (logMAR) & Lens status (P/PS/A, $n$ ) & Combined surgery (n) \\
\hline Macular hole & 10 & $1.09 \pm 0.79$ & $\begin{array}{c}0.75 \pm 0.47 \\
(p=0.17)\end{array}$ & $10 / 0 / 0$ & 6 phaco-IOL \\
\hline PDR with VH & 9 & $2.72 \pm 0.69$ & $\begin{array}{c}1.47 \pm 1.08 \\
(p=0.04)\end{array}$ & $7 / 2 / 0$ & 5 phaco-IOL \\
\hline PDR with TRD & 5 & $1.70 \pm 0.69$ & $\begin{array}{c}1.06 \pm 0.61 \\
(p=0.19)\end{array}$ & $4 / 1 / 0$ & - \\
\hline Endophthalmitis & 3 & $2.43 \pm 0.98$ & $\begin{array}{c}1.86 \pm 0.98 \\
(p=0.42)\end{array}$ & $2 / 1 / 0$ & 1 phaco-IOL \\
\hline RRD & 15 & $1.66 \pm 1.18$ & $\begin{array}{c}1.08 \pm 0.85 \\
(p=0.04)\end{array}$ & $11 / 4 / 0$ & 4 phaco-IOL \\
\hline Perforating injury & 2 & 3.0 & 3.0 & $1 / 0 / 1$ & - \\
\hline DME & 5 & $1.17 \pm 0.56$ & $\begin{array}{c}1.06 \pm 0.65 \\
(p=0.39)\end{array}$ & $4 / 1$ & 1 phaco-IOL \\
\hline Malignant glaucoma & 1 & 0.70 & 0.54 & $0 / 1 / 0$ & - \\
\hline
\end{tabular}

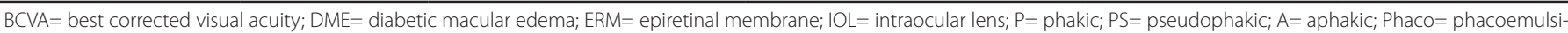
fication; Pre= preoperative; Post= postoperative; $P D R=$ proliferative diabetic retinopathy; $R R D=$ rhegmatogenous retinal detachment; $T R D=$ tractional retinal detachment; $V H=$ vitreous hemorrhage; $\mathrm{VMT}=$ vitreomacular traction. 
Suturing was not required in $60 \%$ of sclerotomies, and the remaining cases were closed with a single transconjunctival-scleral suture. Transient postoperative hypotony (IOP $<6 \mathrm{mmHg}$ ) occurred in two eyes (2.9\%). Increased IOP was observed in four eyes and controlled with antiglaucoma medications. Fibrin formation occurred in eight eyes (11.9\%). Cataract progression was observed in six eyes and managed with phacoemulsification and intraocular lens implantation. Early postoperative $\mathrm{VH}$ occurred in two eyes. Recurrent RD developed in three eyes and were reattached with repeated vitrectomy. Transient intraoperative choroidal detachment was observed in one eye during complicated RD surgery and intraoperatively managed. No other significant complications, such as endophthalmitis; phthisis; or neovascular glaucoma, were observed.

Complicated situations during vitreoretinal surgery can be easily mastered by bimanual techniques than with conventional single-handed methods ${ }^{(3)}$. Our technique using two superior $20-\mathrm{G}$ sclerotomies for a vitrectomy probe allows bimanual surgery to be performed from a more effective approach in complicated cases.

Sutureless sclerotomy along with postoperative hypotony has been postulated to contribute to the potential risk of endophthalmitis following TSV ${ }^{(4)}$. In the present study, 20-G TSV sclerotomy ports were sutured to prevent leakage. Transient postoperative hypotony occurred in two patients, and postoperative endophthalmitis was not observed in any patients.

In a previous study, the rate of early $\mathrm{VH}$ was $11.4 \%$ in patients with proliferative diabetic retinopathy $(\mathrm{PDR})^{(5)}$. In our study, early postoperative $\mathrm{VH}$ was observed in two eyes (2.9\%), however, neither of these cases had a documented history of PDR.
In conclusion, a combined 20- and 23-G vitrectomy technique provides a feasible option in complicated cases with a reasonable complication rate. Future studies are required to evaluate the long-term effects of this procedure, particularly in complicated cases.

Yüksel Totan ${ }^{1}$ Emre Güler ${ }^{2}$

Submitted for publication: April 15, 2015

Accepted for publication: May 5, 2015

${ }^{1}$ Department of Ophthalmology, Medical School, Turgut Özal University, Ankara, Turkey. ${ }^{2}$ Department of Ophthalmology, Erciş State Hospital, Van, Turkey.

Funding: No specific financial support was available for this study.

Disclosure of potential conflicts of interest: None of the authors have any potential conflicts of interest to disclose.

Corresponding author: Emre Güler. Van Yolu Cad. № 57 - Erciş/Van 65400 - Turkey

E-mail: guleremre83@hotmail.com

\section{REFERENCES}

1. Eckardt C. Transconjunctival sutureless 23-gauge vitrectomy. Retina. 2005;25(2):208-11.

2. Spirn MJ. Comparison of 25, 23 and 20-gauge vitrectomy. Curr Opin Ophthalmol. 2009; 20(3):195-9.

3. Eckardt C. Twin lights: a new chandelier illumination for bimanual surgery. Retina 2003:23(6):893-4.

4. Taban M, Ufret-Vincenty RL, Sears JE. Endophthalmitis after 25-gauge transconjunctival sutureless vitrectomy. Retina. 2006;26(7):830-1.

5. Park DH, Shin JP, Kim SY. Comparison of clinical outcomes between 23-gauge and 20-gauge vitrectomy in patients with proliferative diabetic retinopathy. Retina. 2010; 30(10):1662-70

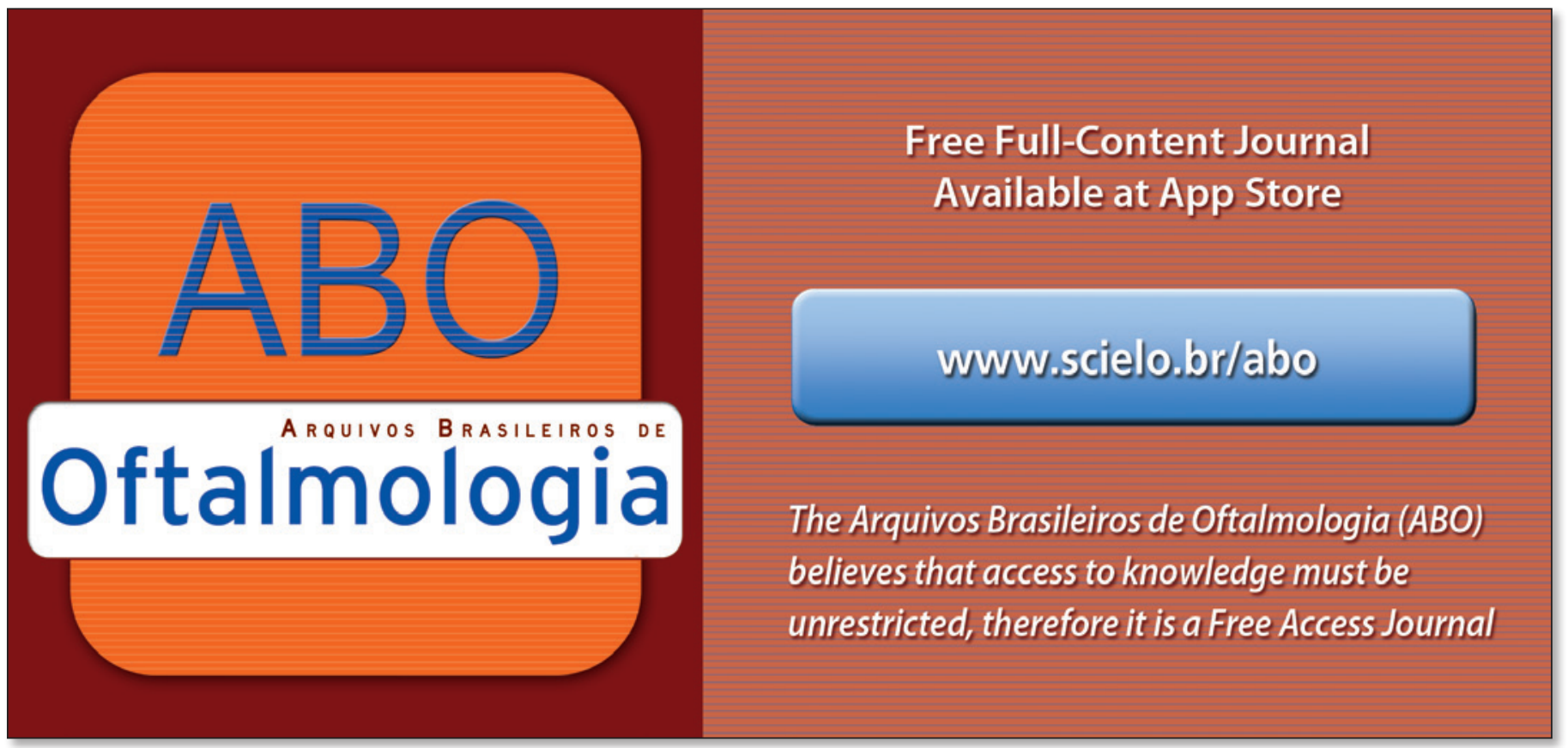

